\title{
Resonance structure in low-energy electron scattering from OCS
}

\author{
T H Hoffmann ${ }^{1}$, H Hotop ${ }^{1}$ and M Allan ${ }^{2}$ \\ ${ }^{1}$ Fachbereich Physik, Technische Universität KL, 67663 Kaiserslautern, Germany \\ ${ }^{2}$ Department of Chemistry, University of Fribourg, 1700 Fribourg, Switzerland
}

\begin{abstract}
Energy-dependent absolute angle-differential cross sections $\left(\theta=135^{\circ}\right)$ for elastic and vibrationally inelastic electron scattering from OCS molecules have been investigated at high resolution (13 meV). The elastic cross section, reported over the range $E=0.06-20 \mathrm{eV}$, exhibits a deep Ramsauer-Townsend minimum near $0.55 \mathrm{eV}$ and the $\pi^{*}(1.2 \mathrm{eV})$ and $\sigma^{*}(3.8 \mathrm{eV})$ resonances known from earlier experimental and theoretical work. The excitation functions for vibrational excitation (VE) of the fundamental CS stretch $(100)$, the CO stretch $\left(\begin{array}{ll}0 & 0\end{array}\right)$ as well as for the fundamental and harmonics of the bending $(0 n 0)$ mode are reported from threshold up to $E=8 \mathrm{eV}$. VE is mediated-in part in a mode-selective way-by threshold peaks and the higher lying resonances. The selectivity in the threshold region can be rationalized in terms of symmetry arguments where the ${ }^{2} \Sigma$ virtual state excites preferentially the $\sigma$ vibrational states with even quanta of bending vibration over the $\pi$ vibrational states with odd quanta of bending vibration. The $\sigma_{\mathrm{CS}}^{*}$ resonance is essentially inactive in VE of the $\mathrm{CO}$ stretch and the bending mode.
\end{abstract}

\section{Introduction}

The series of related triatomic linear molecules $\mathrm{CS}_{2}$, OCS and $\mathrm{CO}_{2}$ is of fundamental interest in connection with lowenergy electron scattering. It allows for systematic studies of, e.g., the influence of the molecular polarizability whose average value increases from $19.6 \mathrm{au}\left(\mathrm{CO}_{2}\right)$ via $38.5 \mathrm{au}$ (OCS) to 59.8 au $\left(\mathrm{CS}_{2}\right)$ [1]. OCS as the intermediate case is the only one to possess a dipole moment of $0.715 \mathrm{D}$ [2]. The vertical electron affinity (VEA) at the equilibrium bending angle $\alpha=180^{\circ}$ strongly increases from $\mathrm{CO}_{2}$ to $\mathrm{CS}_{2}$; for the latter, calculations $[3,4]$ indicate a slightly positive value of the VEA. The calculated adiabatic electron affinities (AEA) range from $-0.54 \mathrm{eV}\left(\mathrm{CO}_{2}, \alpha \approx 138^{\circ}\right)$ via $-0.01 \mathrm{eV}(\mathrm{OCS}$, $\left.\alpha \approx 136^{\circ}\right)$ to $0.46 \mathrm{eV}\left(\mathrm{CS}_{2}, \alpha \approx 143^{\circ}\right)$ [4].

Both scattering theory (numerous studies, see for example $[5,6]$ ) and measurements of total and backward cross sections at very low energies [7] indicate that electron scattering from $\mathrm{CO}_{2}$ at low energies is dominated by a virtual state, which also causes large threshold peaks in the vibrational excitation (VE) cross sections (see, e.g., [8,9]). Calculations of the potential energy surfaces in the region of geometries where the electron is bound (in the fixed-nuclei picture) $[4,10,11]$ reveal that the anion potential surface bends down before crossing the potential of the neutral molecule. This downward-bent section of the potential surface, where an electron is weakly bound to a bent $\mathrm{CO}_{2}$ by a combination of dipole and polarization forces, supports vibrational Feshbach resonances (VFRs) manifested as narrow structures in VE cross sections [12-14], strongly reminiscent of those found in the prototype VFR case of HF [15-17].

For $\mathrm{CS}_{2}$ giant resonances were observed in the total and backward cross sections at very low energies [18]. The VE cross sections are very large in magnitude and exhibit narrow vibrational structure which may be attributed to vibrational levels of the bound anion state [19].

The potential surface for $\mathrm{OCS}^{-}$bends down when approaching the crossing with the neutral OCS surface in a way similar to $\mathrm{CO}_{2}^{-}$[4], indicating the possibility for similar vibrational resonances to occur at energies around $0.2 \mathrm{eV}$. Recent studies of the total scattering cross sections of OCS at energies from 0.07 to $0.3 \mathrm{eV}$, however, have shown no vibrational structure [20].

In this connection, it is of interest to mention recent observations of anion formation in low-energy electron interactions with small clusters $(\mathrm{XY})_{N}$ of $\mathrm{XY}=\mathrm{CO}_{2}$, OCS 
and $\mathrm{CS}_{2}$. Rather sharp VFRs were observed for $\mathrm{CO}_{2}$ clusters $(N \geqslant 4)$ and OCS clusters $(N \geqslant 1)$ while no such structure was observed for $\mathrm{CS}_{2}$ clusters. These results were interpreted to reflect the effects of solvation on the molecular anion state immersed in the cluster environment $[4,21]$.

In this paper, we concentrate on elastic and vibrationally inelastic electron scattering by the molecule OCS at energies below $E=10 \mathrm{eV}$. An overview of previous work up to the year 2000 has been given by Karwasz et al [22], and we refer the reader to references in this review with regard to earlier work. The total cross section, studied before at energies above $0.2 \mathrm{eV}$, is dominated by the $\pi^{*}$ resonance, peaking at about $1.2 \mathrm{eV}$; it is active in mediating VE [23] and dissociative electron attachment (DEA) [23-26]. A Ramsauer-Townsend (RT) minimum has been predicted to occur in the $\sigma$ elastic scattering channel near $0.7 \mathrm{eV}$ in theoretical work [27, 28]. We note that the energy dependence of the elastic differential cross section (DCS), reported by Sohn et al over the range $0.2-5 \mathrm{eV}$ at $130^{\circ}$ (see figure 1 in [29]) exhibits a rather pronounced minimum at $E=0.6 \mathrm{eV}$, but was not addressed as reflecting a RT minimum by these authors. VE was studied by Sohn et al ( $E=0.3-5 \mathrm{eV})$ [29], Abouaf et al $(E=0.15-2 \mathrm{eV})$ [23] and Kawada et al [30]. Of most relevance to the present paper are the results of Abouaf et al obtained with a resolution of $0.025 \mathrm{eV}$. These authors reported excitation functions for the $(0 n 0 ; n=4,5,6)$ bending modes at $\theta=90^{\circ}$ and energies up to $2.5 \mathrm{eV}$ which showed the $\pi^{*}$ resonance at $1.2-1.3 \mathrm{eV}$ and threshold peaks (notably for $n=4,6$ ); they interpreted the latter observation as indicating that a virtual state contributes to the scattering, as confirmed by the later theoretical work $[27,31]$. It is of obvious interest, as noted in [23], to also measure the VE functions for the lower $(0 n 0, n=1-3)$ bending modes to check for the importance of threshold peaks.

In the present work, we reinvestigated low-energy electron scattering from OCS molecules at improved energy resolution (13 meV) with two major aims: (i) search for structure and systematic behaviour in the elastic and vibrationally inelastic scattering channels; (ii) determination of accurate absolute cross sections for these two channels over a range of incident energies. The measurements were limited to one detection angle $\left(135^{\circ}\right)$, chosen such that the effects of the permanent dipole moment — which dominate at small scattering angles-are suppressed. Using previously published angular distributions, our absolute differential cross sections can be used to establish integral cross sections at selected energies. The paper is organized as follows. In section 2, we briefly describe the experimental setup and procedure. In section 3, we present and discuss the experimental results. We conclude with a short summary.

\section{Experiment}

Electrons emitted from a hot filament are energy-selected by a double hemispherical monochromator and focused onto an effusive beam target. A double hemispherical analyser allows for detection of elastically or inelastically scattered electrons. Further details of the experiment and procedures have been

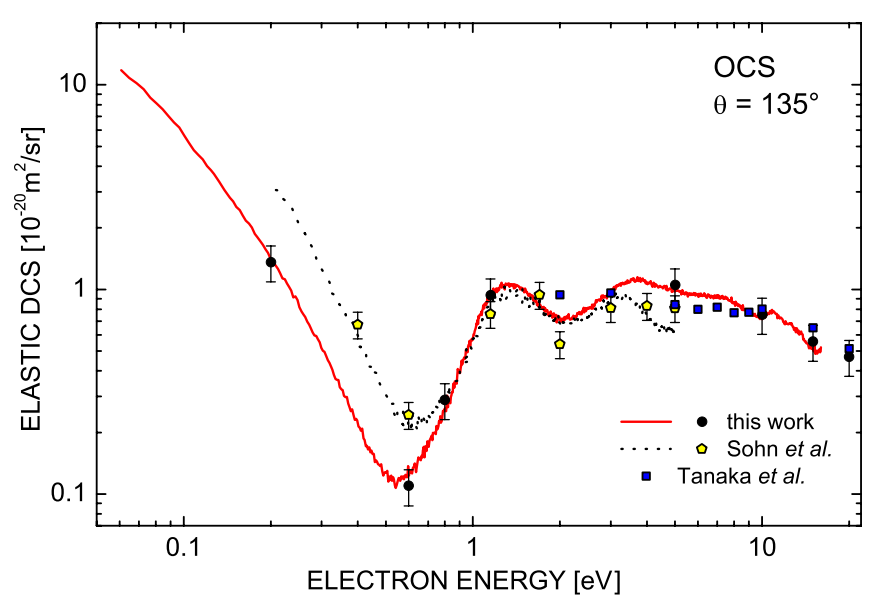

Figure 1. Elastic cross section at an angle of $135^{\circ}$, shown as a function of electron energy with absolute values indicated by filled circles. The data of Sohn et al [29] are shown for comparison-their excitation function at $130^{\circ}$ by dots, their tabulated values, interpolated between $130^{\circ}$ and $138^{\circ}$, by empty pentagons with error bars. Depicted as squares are the results of Tanaka and Hoshino [36], measured at an angle of $130^{\circ}$.

Table 1. Differential elastic cross sections $\left(10^{-20} \mathrm{~m}^{2} \mathrm{sr}^{-1}\right)$ at $135^{\circ}$.

\begin{tabular}{llllllccc}
\hline Energy $(\mathrm{eV})$ & 0.2 & 0.6 & 0.8 & 1.15 & 5.0 & 10.0 & 15.0 & 20.0 \\
DCS & 1.36 & 0.11 & 0.29 & 0.94 & 1.05 & 0.75 & 0.56 & 0.47 \\
\hline
\end{tabular}

described elsewhere [32-34]. Absolute elastic cross sections were measured by comparison with the known helium cross sections using the relative flow method [35]. The pressure ratio between $\mathrm{He}$ and OCS was chosen as roughly 2.4 according to their molecular diameters, in order to get the same beam profile. The flow rates used were $0.106-0.136 \mathrm{mbar} \mathrm{cm}^{3} \mathrm{~s}^{-1}$ for OCS at pressures of 0.075-0.092 mbar and 0.892$1.294 \mathrm{mbar} \mathrm{cm}^{3} \mathrm{~s}^{-1}$ at $0.172-0.243 \mathrm{mbar}$ for He. The measurement procedure compensated for small variations of pressure and beam current during the signal accumulation. The confidence limit ( 2 standard deviations) is about $\pm 20 \%$ for the elastic cross sections and $\pm 25 \%$ for the vibrationally inelastic cross sections. The incident electron resolution was about $13 \mathrm{meV}$ with a beam current of about $40 \mathrm{pA}$, due to the low electron energies and deposits on the electron optics, which resulted in the frequent need for readjustment of the electron gun. The sample was introduced through a $0.25 \mathrm{~mm}$ diameter nozzle kept at about $30^{\circ} \mathrm{C}$.

\section{Results and discussion}

\subsection{Elastic scattering}

Absolute elastic cross sections were measured at eight different energies, and are shown by full circles in figure 1 and listed in table 1. The elastic signal was then measured as a function of the incident electron energy, corrected for the variation of the instrumental response function, normalized to the discrete absolute measurement at $1.15 \mathrm{eV}$, and is shown by a continuous line in figure 1. 


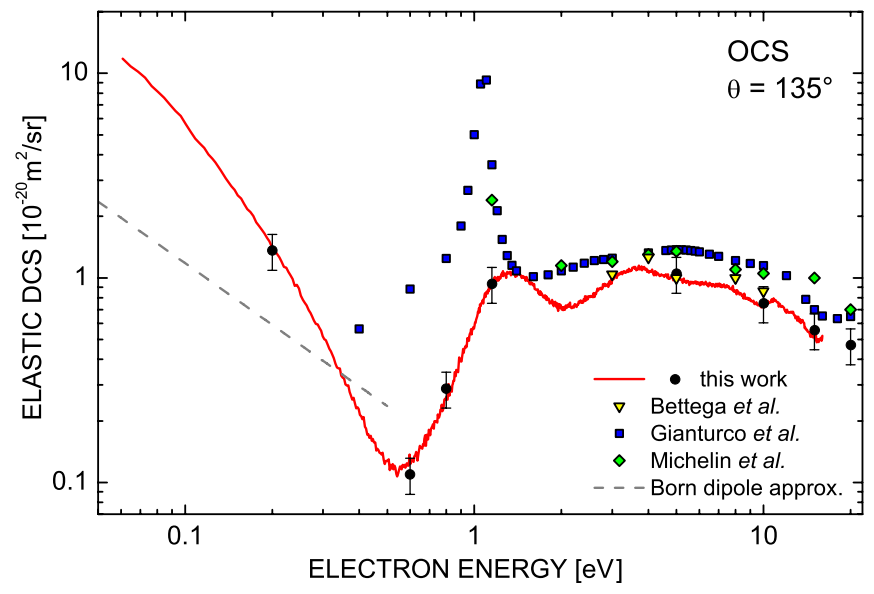

Figure 2. Elastic cross section at an angle of $135^{\circ}$, shown as a function of electron energy with absolute values indicated by filled circles. Theoretical data by Bettega et al [31] are indicated by open triangles, calculated cross sections by Gianturco and Stoecklin [28] are depicted as squares and results from Michelin et al [37] are shown as diamonds. A Born approximation calculation for scattering from a polar molecule [38] is indicated by the dashed line.

The present results are in excellent agreement with the data of Sohn et al [29] in the range $0.8-3 \mathrm{eV}$, but larger above about $3 \mathrm{eV}$. At energies below about $0.7 \mathrm{eV}$ the present cross sections are generally smaller and the minimum at $0.55 \mathrm{eV}$ appears at slightly lower energy. The apparent depth of the RT minimum is a function of resolution and the present improved resolution leads to a deeper dip in the signal. The resolution (and possibly also a small difference in energyscale calibration) are also the probable cause of the difference in the cross section magnitude below $0.7 \mathrm{eV}$ - at a given energy ( $0.3 \mathrm{eV}$ as an example), an incident beam with lower resolution contains more slow electrons, and leads to a larger apparent cross section in a situation where the cross section rises rapidly with decreasing energy.

In figure 2 we compare our elastic cross section with theoretical results. The present data are in very good agreement with a static-exchange plus polarization Schwinger multichannel calculation of Bettega et al [31] at 3, 4, 5 and $8 \mathrm{eV}$. Good agreement is also found with the cross sections of Gianturco and Stoecklin [28] at energies of 1.5-20 eV, while their values at lower energies are larger than the present experimental data. Gianturco and Stoecklin employed a separable representation of the exchange potential and added correlation-polarization effects via a density functional formulation. We also include the cross sections of Michelin et al [37] involving the Schwinger variational iterative method, combined with a distorted wave approximation; their values are somewhat larger than the present experiment, similarly to those of Gianturco and Stoecklin. Also indicated in the figure is the rotationally inelastic cross section for scattering from a fixed molecule with dipole moment, averaged over orientation, as given by Altshuler (see equation (22) in [38]).

The dramatic increase of the cross section towards low energies is attributed to a virtual state [27, 31], the deep minimum around $0.55 \mathrm{eV}$ is attributed to the RamsauerTownsend effect [27, 28]. The fact that the measured

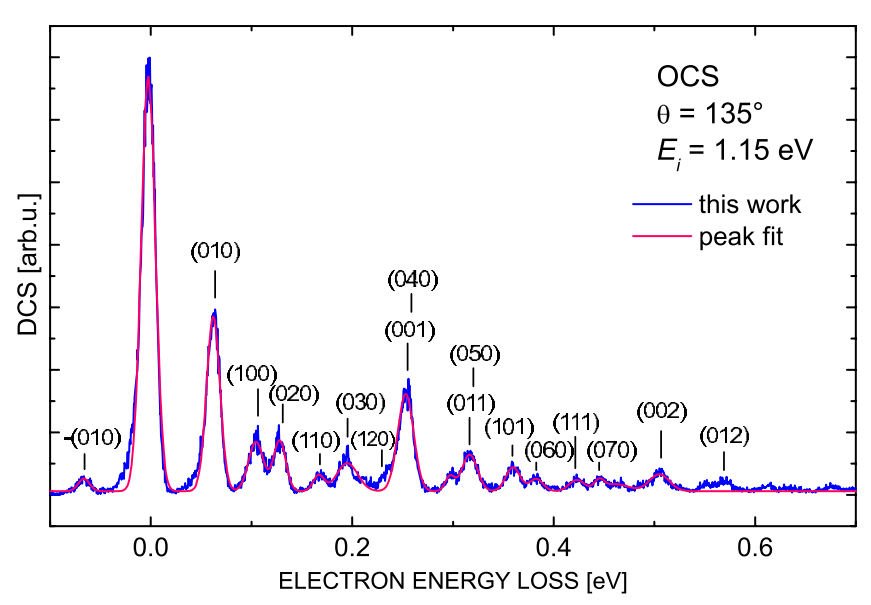

Figure 3. Spectrum with constant incident energy at an angle of $135^{\circ}$. The ordinate scale is linear.

Table 2. Summary of vibrational energies [39] and dipole transition matrix elements [40] in OCS.

\begin{tabular}{lllll}
\hline No & Type & Symm. & Energy $(\mathrm{meV})$ & $D(\mathrm{au})$ \\
\hline$v_{1}$ & CS str & $\sigma^{+}$ & 106.5 & 0.0243 \\
$\nu_{2}$ & Bend & $\pi$ & 64.5 & 0.0185 \\
$\nu_{3}$ & CO str & $\sigma^{+}$ & 255.6 & 0.134 \\
\hline
\end{tabular}

cross section is much larger than predicted by the Born approximation below $200 \mathrm{meV}$ is taken as a manifestation of the virtual state, the fact that it is lower above about $350 \mathrm{meV}$ as a consequence of the Ramsauer-Townsend minimum.

The peak at $1.33 \mathrm{eV}$ has been assigned to $\mathrm{a}^{2} \Pi$ resonance, that at $3.7 \mathrm{eV}$ to overlapping ${ }^{2} \Sigma$ and ${ }^{2} \Delta$ resonances, with the former being dominant [29]. This assignment of the resonances is compatible with the symmetry decomposition of the calculated integral cross section of Bettega et al [31] and of Gianturco and Stoecklin [28]. The latter group also calculated resonances at higher energies, $\mathrm{a}^{2} \Pi$ resonance around $10 \mathrm{eV}$, which could correspond to the weak peak at $10.8 \mathrm{eV}$ in our data in figure 1.

\subsection{Vibrational excitation}

The fundamental vibrations of OCS are summarized in table 2. ${ }^{3}$ Absolute values of the cross sections for vibrational excitation were determined from the energy-loss spectrum shown in figure 3 , recorded with a constant incident energy of $1.15 \mathrm{eV}$, near the centre of the ${ }^{2} \Pi$ resonance. The spectrum was corrected for the variation of the analyser response function and the area under the elastic peak was normalized to the measured absolute value. Peak overlap was partially resolved by fitting the observed spectrum to Gaussian profiles. The results are given in table 3 . The spectrum is characteristic for vibrational excitation by $\mathrm{a}^{2} \Pi$ resonance, as has already been pointed out in earlier work [23, 29]. The VE cross sections are large, the sum under all vibrationally inelastic peaks (up to an energy loss of $1 \mathrm{eV}$ ) amounts to $1.7 \times 10^{-20} \mathrm{~m}^{2} \mathrm{sr}^{-1}$, i.e.

3 We adopted the notation of Bishop and Cheung [40]. In earlier works, the numbers assigned to $\mathrm{CS}$ stretch and $\mathrm{CO}$ stretch were interchanged $[39,50]$. 


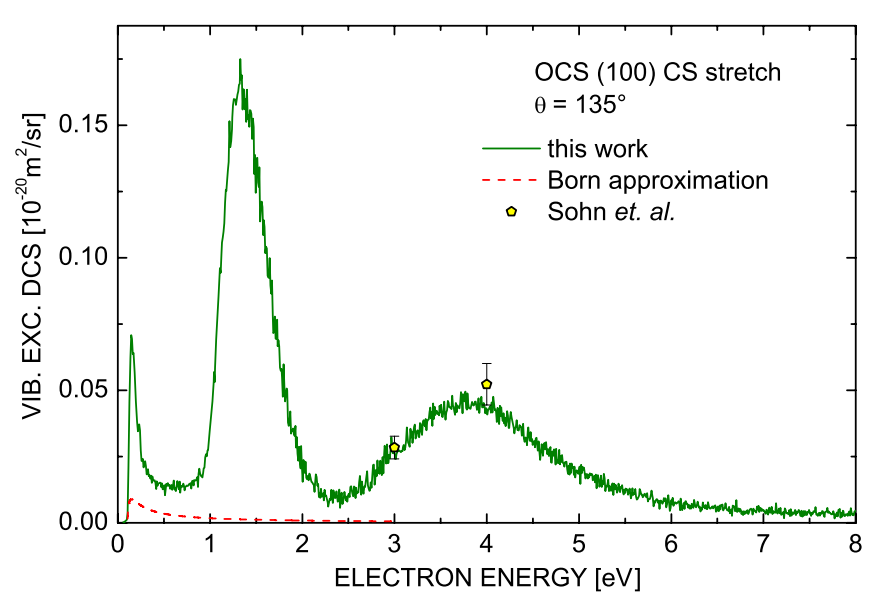

Figure 4. Differential cross section for excitation of the CS stretch vibration at an angle of $135^{\circ}$.

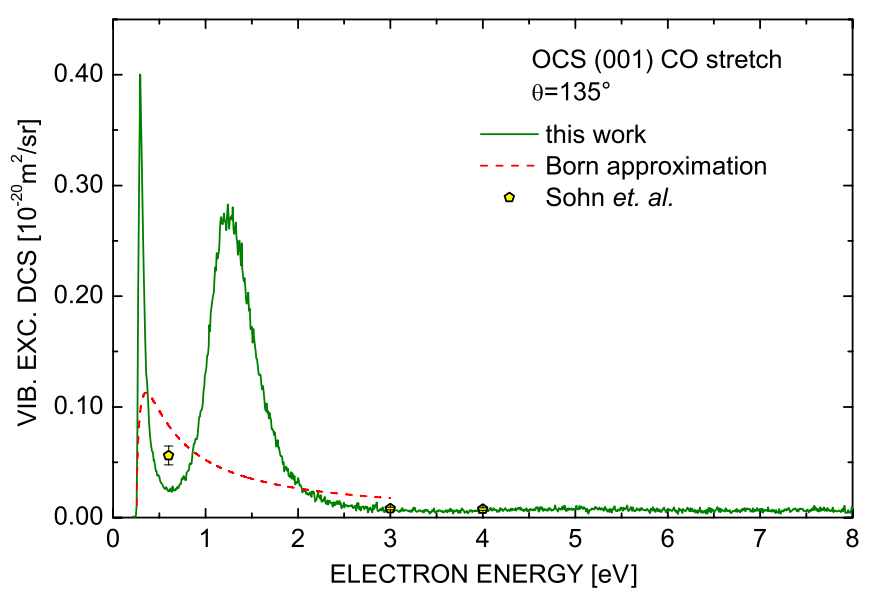

Figure 5. Differential cross section for excitation of the CO stretch vibration at an angle of $135^{\circ}$. Note that it is degenerate with four quanta of the bending vibration.

Table 3. Differential VE cross sections $\left(10^{-20} \mathrm{~m}^{2} \mathrm{sr}^{-1}\right)$ at $E_{i}=$ $1.15 \mathrm{eV}$ and $135^{\circ}$.

\begin{tabular}{llllll}
\hline Final state & $(010)$ & $(020)$ & $(030)$ & $(100)$ & $(001)$ \\
DCS & 0.38 & 0.11 & 0.076 & 0.11 & 0.26 \\
\hline
\end{tabular}

1.8 times the elastic cross section, which is $0.94 \times$ $10^{-20} \mathrm{~m}^{2} \mathrm{sr}^{-1}$ (including rotational transitions). Not only the fundamentals but also the overtones of all three vibrations, as well as many combination vibrations are excited, a consequence of the fact that the temporary occupation of the $\pi^{*}$ orbital lengthens both the CS and the CO bonds and makes the $\mathrm{OCS}^{-}$bent.

The cross sections for exciting specific vibrations as a function of electron energy were then obtained by recording the respective excitation functions, correcting them for the instrumental response function and normalizing them to the absolute values given in table 3 . The results are shown in figures 4-6. The agreement with the individual data points of Sohn et al [29] is satisfactory. The contribution of direct scattering to vibrational excitation can be estimated using first Born approximation [41] and dipole transition matrix elements determined by infrared spectroscopy [40]. Though the results at high scattering angles will not be quantitative, they allow

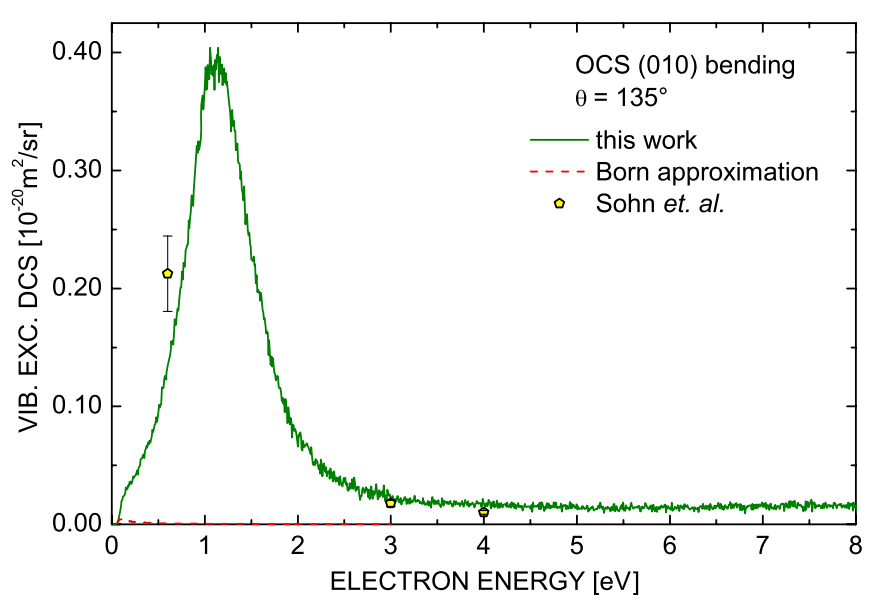

Figure 6. Differential cross section for excitation of the bending vibration at an angle of $135^{\circ}$.

for a survey of the different contributions to threshold peaks [42], and are shown as dashed lines in figures 4-6.

The cross section for the excitation of the CS stretch mode $\left(\begin{array}{lll}1 & 0 & 0\end{array}\right)$ in figure 4 shows a considerable threshold peak and two shape resonances, one around $1.3 \mathrm{eV}$ and a second around $3.8 \mathrm{eV}$. As already discussed in connection with the elastic cross section, they can be assigned as $^{2} \Pi$ and ${ }^{2} \Sigma[28,31,43]$. The threshold peak must be primarily due to the virtual state since only a small fraction of it is explained by the Born approximation.

The CO stretch mode is nearly degenerate with four quanta of the bending mode (energy difference around $5 \mathrm{meV}$ [44]), cannot be resolved in the present experiment, and figure 5 consequently shows the sum of the cross sections for exciting both the (0 01$)$ and the ( 040$)$ states. Both a threshold peak and the ${ }^{2} \Pi$ band are observed. The threshold peak is narrower than the Born prediction, but the Born prediction is not expected to be very reliable at large scattering angles. Interestingly the ${ }^{2} \Sigma$ resonance is practically absent, indicating that the temporarily occupied $\sigma^{*}$ orbital is localized on, and antibonding with respect to, the CS bond and has nearly no coefficients on the $\mathrm{CO}$ bond.

The excitation function for the bending mode (figure 6) does not exhibit a threshold peak and this aspect will be discussed further below. The ${ }^{2} \Pi$ resonance is prominent, consistent with its bent equilibrium geometry. The ${ }^{2} \Sigma$ resonance is absent, indicating a linear equilibrium geometry, consistent with its assignment.

Sohn et al [29] and Abouaf et al [23] already reported a preference for exciting even quanta of the bending vibration over odd quanta. We further study this effect by recording the cross sections for exciting the $(0 n 0)$ states for $n$ up to 8 , as shown in figure 7 . Some of the spectra are affected by band overlap, in particular the (080) and the $\left(\begin{array}{lll}0 & 0 & 2\end{array}\right)$ states as already mentioned above. Clear trends in the spectra emerge, despite this limitation:

(i) The relative intensity of the threshold peak over the ${ }^{2} \Pi$ resonant peak diminishes with increasing $n$. This is consistent with the excitation process near threshold 


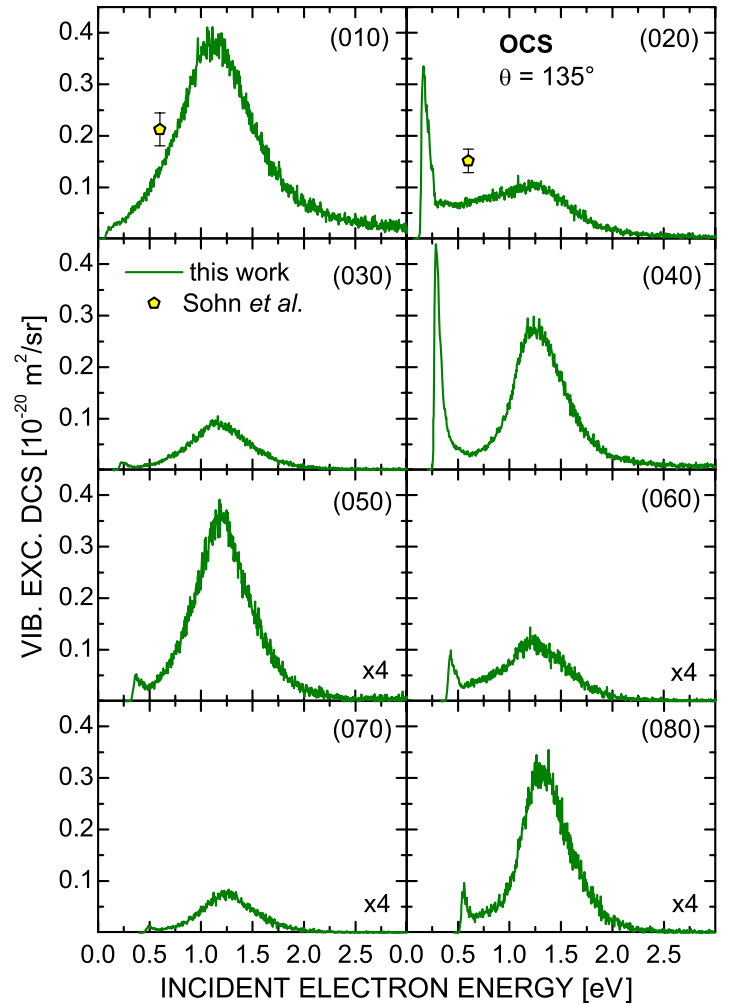

Figure 7. Excitation of different harmonics of the bending vibration at an angle of $135^{\circ}$.

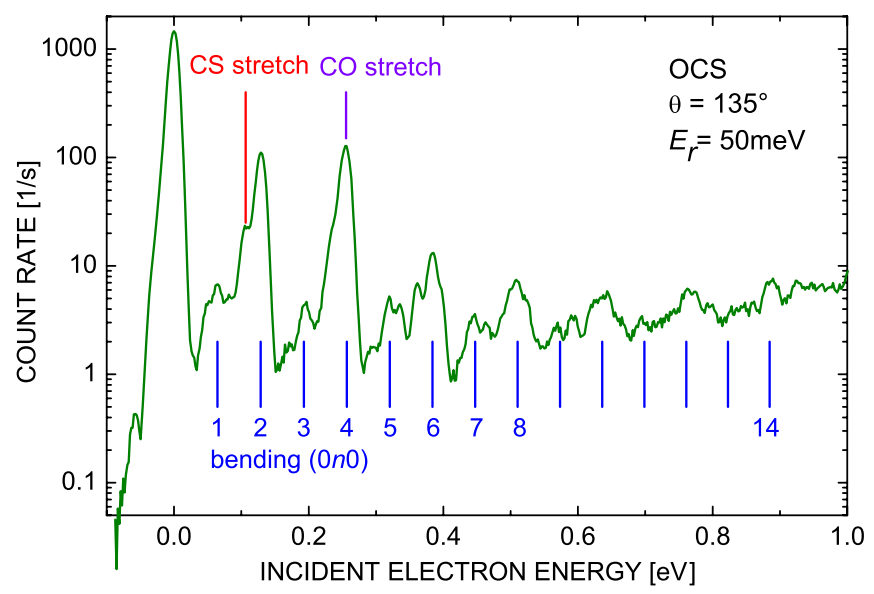

Figure 8. Threshold excitation spectrum at an angle of $135^{\circ}$.

having a greater autodetachment width than the ${ }^{2} \Pi$ resonance.

(ii) For every pair $(0, n, 0)$ and $(0, n+1,0)$, left and right in figure 7 , the threshold peak is higher on the right, for the excitation of even number of bending quanta.

(iii) The difference in the height of the threshold peaks between even and odd $n$ is largest for low $n$. The threshold peak is entirely absent for (010), and completely dominates the spectrum for $(020)$. The difference is smaller but still very clear between $(070)$ and $(080)$.

Another way of visualizing the relative intensities of the threshold peaks in vibrational excitation is by means of an energy-loss spectrum recorded with a low constant residual energy, such as the spectrum recorded at $E_{r}=0.05 \mathrm{eV}$ shown in figure 8. (A very similar spectrum, recorded at $90^{\circ}$ (for energies up to $2 \mathrm{eV}$ with $E_{r} \approx 0 \mathrm{eV}$ ), was presented by Abouaf et al [23].) The alternation of the intensities of the $(0 n 0)$ peaks, with even $n$ being consistently higher than odd $n$, is clearly visible. Note that the vertical scale is logarithmic and the enhancement of even $n$ over odd $n$ is generally more than a factor of 2 .

Schulz and Wong [45] observed selectivity in vibrational excitation of a polyatomic molecule (benzene) and derived a selection rule to explain it. (A further discussion was given by Gallup [46, 47] and by Ben Arfa and Tronc [48].) They proposed that the vibrational state must have the same symmetry as the force field exciting it, that is, for our case of $\mathrm{a}^{2} \Pi$ resonance, $\Pi \otimes \Pi=\Sigma(+\Delta)$. This selection rule would thus not allow the excitation of odd quanta of the bending vibration, having $\pi$ symmetry (neglecting angular momenta higher than 2 throughout). Schulz and Wong observed more vibrations than predicted by this selection rule, however. They were able to explain the additional vibrations by assuming that the electron may change angular momentum upon exciting a vibration, in particular that it can leave in an s-wave. In our case of $\mathrm{a}^{2} \Pi$ resonance this adds vibrations of the $\Pi \otimes \Sigma=\Pi$ symmetry, i.e., the excitation of odd quanta also becomes allowed. The extended selection rule appears applicable to OCS because no clear preference for even quanta of bending is apparent in the ${ }^{2} \Pi$ resonance region, both in figure 3 and in figure 7. (There is at least one case, however, where a ${ }^{2} \Pi$ resonance leads to preferential excitation of even quanta of a bending vibration - that of diacetylene [49].) Abouaf et al [23] have shown both experimentally and theoretically that the angular distributions of the excitation of the odd and even quanta are quite different, however.

In the near-threshold region, dominated by the ${ }^{2} \Sigma$ virtual state, the above rules predict that only $\sigma$ vibrational states will be excited, i.e., only even quanta of the bending vibration (and, of course, the CS and CO stretch vibrations). The selection rule thus explains the observations.

In view of these results it is interesting to pose the question of whether this selectivity is also manifest in the remaining two members of the present series, $\mathrm{CO}_{2}$ and $\mathrm{CS}_{2}$. The excitation of the $(0,1,0)$ state in $\mathrm{CO}_{2}$ gives rise to the strongest vibrational peak in a spectrum recorded near threshold $[8,12]$, in an apparent violation of the symmetry rule, but it has been argued that the observed cross section (at $54^{\circ}$ ) can be entirely attributed to direct dipole excitation [8]. The excitation of the next odd number of quanta, $(0,3,0)$, is also (weakly) observed in $\mathrm{CO}_{2}$, although the situation is complicated by the fact that it is mixed with the $(1,1,0)$ state by a Fermi resonance [12]. The problem has also been addressed by Sommerfeld et al [11]. It is thus difficult to decide whether the symmetryinduced intensity alternance of bending excitation occurs in $\mathrm{CO}_{2}$. Vibrational states with odd quanta of bending vibration are readily excited in $\mathrm{CS}_{2}$ near the threshold [19], but this is less surprising, because even the vertical electron affinity of $\mathrm{CS}_{2}$ is positive and the ${ }^{2} \Pi_{\mathrm{u}}$ state probably dominates VE even at the threshold. 
The last question which this study addresses is that of the possible existence of sharp structures in the cross sections at low energies. As already mentioned in the introduction, such structures could be expected, based on several arguments:

(i) Sharp structures were observed in OCS clusters [4], less pronounced but otherwise similar to those found in $\mathrm{CO}_{2}$ clusters.

(ii) Sharp structures were observed for isolated $\mathrm{CO}_{2}$ molecules, in the excitation of the topmost members of Fermi polyads containing overtones (at least two quanta) of the symmetric stretch vibrations [12].

(iii) The structures in $\mathrm{CO}_{2}$ are believed to be linked to a weak binding of an electron by a combination of dipole and polarization forces in the bend geometry, which causes the potential surface of the anion to bend down before crossing the potential of the neutral molecule $[4,10,11]$. This binding may be expected to be even stronger in OCS because of its larger polarizability and permanent dipole moment.

None of the curves in figures 4-7 exhibits structures near higher excited thresholds, however. Note that the third overtone of the CS stretch vibration, (300), overlaps with the fifth overtone of bending, (050), shown in figure 7, and any structure in the (300) cross section would be visible there. The second overtone of the $\mathrm{CO}$ stretch vibration, $\left(\begin{array}{ll}0 & 0\end{array}\right)$, is close to the $(080)$ vibration, also shown in figure 7 . No structures are thus observed, not even in the overtones of any of the three vibrational modes.

With this negative result in mind, we note that, according to the calculations [4], the potential surface of $\mathrm{OCS}^{-}$bends down, but less than that of $\mathrm{CO}_{2}$, despite the larger polarizability of OCS. This result could explain the absence of structure in the cross sections. The fact that the structures in the OCS clusters are less pronounced than those in $\mathrm{CO}_{2}$ clusters points in the same direction. Finally, we note that $\mathrm{CO}_{2}$ and OCS differ in two other aspects:

(i) The structures in $\mathrm{CO}_{2}$ appear only in the vibrations containing the symmetric stretch, they are absent in the antisymmetric stretch. This symmetry distinction is missing in OCS.

(ii) Both stretch and bending are important for the stabilization of the $\mathrm{CO}_{2}^{-}$anion, and it is thus important that in the vibrational states for which structure appears in the cross sections, the stretch and bending motions are mixed, in phase, by strong Fermi resonances in $\mathrm{CO}_{2}$ [13]. This aspect is missing in OCS where Fermi mixing is weaker.

\section{Conclusions}

We report absolute cross sections for elastic and vibrationally inelastic electron scattering from OCS molecules, as measured with high energy resolution $(13 \mathrm{meV})$ at the selected angle $\theta=135^{\circ}$ over the energy $0.06-20 \mathrm{eV}$. In the range of overlap, satisfactory to good agreement is obtained with the previous experimental results of Sohn et al [29], Abouaf et al [23] and Tanaka and Hoshino [36] and the theoretical cross sections of
Michelin et al [37], Bettega et al [27, 31] and Gianturco and Stoecklin [28].

More specifically, the present measurement of the elastic cross section confirms earlier measurements between 0.8 and $3 \mathrm{eV}$, but is larger between 3 and $5 \mathrm{eV}$ and lower below $0.8 \mathrm{eV}$. Very good agreement is found with the static-exchange plus polarization approximation results of Bettega et al [31] between 3 and $8 \mathrm{eV}$ and the calculation including correlationpolarization effects of Gianturco and Stoecklin [28] at energies of $1.5-20 \mathrm{eV}$. The present data are lower than the latter calculation below $1.5 \mathrm{eV}$, however.

The magnitudes of the present VE cross sections are in satisfactory agreement with the data of Sohn et al [29]. The $3.7 \mathrm{eV}$ resonance excites selectively the $\mathrm{CS}$ stretch vibration, but not the $\mathrm{CO}$ stretch and the bending vibrations, confirming its assignment as $\sigma_{\mathrm{CS}}^{*}$. The $1.33 \mathrm{eV}$ resonance excites all three modes, consistent with a temporary $\pi^{*}$ occupation with a bent equilibrium geometry. A long progression of bending vibration with a strong preference for even quanta is excited near the threshold, consistent with a ${ }^{2} \Sigma$ virtual state mechanism, which prefers $\sigma$ final vibrational states over $\pi$ states.

Despite thorough search, including overtone vibrations, no sharp resonant structures were found in the VE cross section near higher vibrational thresholds. This is somewhat surprising, because sharp VFRs were found in OCS clusters and because the potential energy surfaces of $\mathrm{OCS}^{-} / \mathrm{OCS}$ resemble those of $\mathrm{CO}_{2}^{-} / \mathrm{CO}_{2}$ [4], and because sharp VFR were found in $\mathrm{CO}_{2}$, both for isolated molecules [12] and in clusters [21].

\section{Acknowledgments}

This work has been supported by the Deutsche Forschungsgemeinschaft through Forschergruppe FOR 307 as well as through grant Ho 427/28, by the Forschungszentrum Optische Technologien und Lasergesteuerte Prozesse, by the European Science Foundation through the network EIPAM (Electron-Induced Processing At the Molecular level), and by the Swiss National Science Foundation (project 200020113599). We gratefully acknowledge $H$ Tanaka and $M$ Hoshino, F A Gianturco and T Stoecklin for providing cross sections in numerical form and I I Fabrikant for helpful discussions. One of us (THH) thanks M Allan, N C Jones and D Field for their hospitality during visits to Fribourg and Aarhus.

\section{References}

[1] Lide D R (ed) 2007 CRC Handbook of Chemistry and Physics 88th edn (Boca Raton, FL: CRC Press)

[2] Tanaka K, Ito H, Harada K and Tanaka T 1984 J. Chem. Phys. 805893

[3] Gutsev G L, Bartlett R J and Compton R N 1998 J. Chem. Phys. 1086756

[4] Barsotti S, Sommerfeld T, Ruf M-W and Hotop H 2004 Int. J. Mass Spectrom. Ion Process. 233181

[5] Estrada H and Domcke W 1985 J. Phys. B: At. Mol. Phys. 184469

[6] Morgan L A 1998 Phys. Rev. Lett. 801873 
[7] Field D, Jones N C, Lunt S L and Ziesel J-P 2001 Phys. Rev. A 64022708

[8] Kochem K, Sohn W, Hebel N, Jung K and Ehrhardt H 1985 J. Phys. B: At. Mol. Phys. 184455

[9] Allan M 2001 Phys. Rev. Lett. 87033201

[10] Sommerfeld T 2003 J. Phys. B: At. Mol. Opt. Phys. 36 L127

[11] Sommerfeld T, Meyer H-D and Cederbaum L S 2004 Phys. Chem. Chem. Phys. 642

[12] Allan M 2002 J. Phys. B: At. Mol. Opt. Phys. 35 L387

[13] Vanroose W, Zhang Z, McCurdy C W and Rescigno T N 2004 Phys. Rev. Lett. 92053201

[14] Rescigno T N, McCurdy C W, Haxton D J, Trevisan C S and Orel A E 2007 J. Phys. Conf. Ser. $\mathbf{8 8} 012027$

[15] Knoth G, Gote M, Rädle M, Jung K and Ehrhardt H 1989 Phys. Rev. Lett. 621735

[16] Č́ízek M, Horáček J, Allan M, Fabrikant I I and Domcke W 2003 J. Phys. B: At. Mol. Opt. Phys. 362837

[17] Hotop H, Ruf M-W, Allan M and Fabrikant I I 2003 Adv. At. Mol. Opt. Phys. 4985

[18] Jones N C, Field D, Ziesel J-P and Field T A 2002 Phys. Rev. Lett. 89093201

[19] Allan M 2003 J. Phys. B: At. Mol. Opt. Phys. 362489

[20] Jones N C, Hoffmann T H and Field D (unpublished results)

[21] Barsotti S, Leber E, Ruf M-W and Hotop H 2002 Int. J. Mass Spectrom. Ion Process. 220313

[22] Karwasz G P, Brusa R S and Zecca A 2001 Riv. Nuovo Cimento 241

[23] Abouaf R, Pommier J, Cvejanovic S and Saubaméa B 1994 Chem. Phys. 188339

[24] Ziesel J P, Schulz G J and Milhaud J 1975 J. Chem. Phys. 621936

[25] Abouaf R and Fiquet-Fayard F 1976 J. Phys. B: At. Mol. Phys. $9 \mathrm{~L} 323$

[26] Tronc M, Malegat L and Azria R 1982 Chem. Phys. Lett. 92551

[27] Bettega M H F, Lima M A P and Ferreira L G 2005 Phys. Rev. A 72014702
[28] Gianturco F A and Stoecklin T 2007 Chem. Phys. 332145

[29] Sohn W, Kochem K-H, Scheuerlein K M, Jung K and Ehrhardt H 1987 J. Phys. B: At. Mol. Phys. 203217

[30] Kawada M K, Sueoka O and Kimura M 2000 J. Chem. Phys. 1127057

[31] Bettega M H F, Lima M A P and Ferreira L G 2004 Phys. Rev. A 70062711

[32] Allan M 1992 J. Phys. B: At. Mol. Opt. Phys. 251559

[33] Allan M 2005 J. Phys. B: At. Mol. Opt. Phys. 383655

[34] Allan M 2007 J. Phys. B: At. Mol. Opt. Phys. 403531

[35] Nickel J C, Zetner P W, Shen G and Trajmar S 1989 J. Phys. E: Sci. Instrum. 22730

[36] Tanaka H and Hoshino M 2008 private communication

[37] Michelin S E, Kroin T, Iga I, Homem M G P, Miglio H S and Lee M T 2000 J. Phys. B: At. Mol. Opt. Phys. 333293

[38] Altshuler S 1957 Phys. Rev. 107114

[39] Shimanouchi T 1972 Tables of Molecular Vibrational Frequencies (NBS Ref. Data Ser. 39 vol I) (Washington, DC: US Govt Printing Office)

[40] Bishop D M and Cheung L M 1982 J. Phys. Chem. Ref. Data 11119

[41] Itikawa Y 2004 J. Phys. B: At. Mol. Opt. Phys. 37 R1

[42] Allan M, Craig N C and McCarty L V 2002 J. Phys. B: At. Mol. Opt. Phys. 35523

[43] Lynch M G, Dill D, Siegel J and Dehmer J L 1979 J. Chem. Phys. 714249

[44] Zúñiga J., Bastida A, Alacid M and Requena A 2000 J. Chem. Phys. 1135695

[45] Wong S F and Schulz G J 1975 Phys. Rev. Lett. 351429

[46] Gallup G A 1986 Phys. Rev. A 342746

[47] Gallup G A 1993 J. Chem. Phys. 99827

[48] Ben Arfa M and Tronc M 1990 J. Electron Spectrosc. Relat. Phenom. 50117

[49] Allan M 1984 Chem. Phys. 86303

[50] Herzberg G 1966 Molecular Spectra and Molecular Structure: III. Electronic Spectra and Electronic Structure of Polyatomic Molecules (New York: Van Nostrand Reinhold) 